\title{
Room-temperature stability of Pt nanogaps formed by self-breaking
}

\author{
F. Prins, ${ }^{1}$ T. Hayashi, ${ }^{2}$ B. J. A. de Vos van Steenwijk, ${ }^{1}$ B. Gao, ${ }^{1}$ E. A. Osorio, ${ }^{1}$ K. Muraki, ${ }^{2}$ \\ and H. S. J. van der Zant ${ }^{1, a)}$ \\ ${ }^{1}$ Kavli Institute of Nanoscience, Delft University of Technology, Lorentzweg 1, 2628 CJ Delft, \\ The Netherlands \\ ${ }^{2}$ NTT Basic Research Laboratories, NTT Corporation, 3-1, Morinosato-Wakamiya, Atsugi, \\ Kanagawa 243-0198, Japan
}

(Received 22 January 2009; accepted 9 March 2009; published online 26 March 2009)

\begin{abstract}
We present a method to make Pt nanometer-spaced electrodes that are free of metallic particles and stable at ambient conditions. The nanogaps are fabricated using feedback-controlled electromigration to form few-atom contacts. When performing this procedure at elevated temperatures $(>420 \mathrm{~K})$, the $\mathrm{Pt}$ contacts undergo self-breaking so that nanometer separated electrode pairs are formed. Once cooled down to lower temperatures, the nanogaps stabilize and can be characterized in detail. We find that current-voltage characteristics can be well fitted to a Simmons model for tunneling and gap-size fluctuations at room temperature determined from these fits stay within $0.6 \AA$ for at least 50 h. (C) 2009 American Institute of Physics.
\end{abstract}

[DOI: 10.1063/1.3109784]

Fabrication of three-terminal device architectures for single-molecular electronics has been performed by electromigration (EM) of thin Au wires. ${ }^{1} \mathrm{EM}$ is the directed migration of atoms by momentum transfer from electrons when a large current density is applied to a wire. This process requires sufficient atomic mobility and is therefore assisted by Joule heating. The planar geometry of the EM devices results in a relatively large gate-to-molecule coupling and great stability at low temperatures for systematic studies as a function of temperature and magnetic field. ${ }^{2}$ However, Au electrodes are not stable at room temperature due to the high mobility of $\mathrm{Au}$ and residual stress on the wire after EM. ${ }^{3}$ As it was shown in Ref. 4, the instability of $\mathrm{Au}$ at room temperature can be used advantageously when wires narrowed by feedback-controlled EM to a few atoms "self-break" without an applied voltage. After formation of the gap, the selfbreaking continues to widen the gap up to several nanometers unless the device is cooled down and the atomic mobility is frozen. The formation of metallic clusters is largely avoided with this method, a problem often observed in traditional EM techniques. 5,6

By performing experiments at different temperatures we show in this letter that self-breaking of $\mathrm{Au}$ and $\mathrm{Pt}$ few-atom contacts follows a temperature activated behavior. The activation barrier is higher for $\mathrm{Pt}$, so that self-breaking is not possible at room temperature but does occur at a temperature roughly $100{ }^{\circ} \mathrm{C}$ higher. Based on these results we have developed a method in which we perform EM and selfbreaking of $\mathrm{Pt}$ nanowires at elevated temperatures after which a cool down to room temperature suffices to freeze the atomic mobility. This process results in nanogaps that are free of metallic clusters and that are stable at room temperature.

Fabrication of the few-atom constrictions is done by feedback-controlled EM of nanowires. ${ }^{7}$ The nanowires are fabricated by evaporating Au or Pt thin wire bridges, with a cross section of $100 \times 13 \mathrm{~nm}^{2}$ and a length of $300 \mathrm{~nm}$.

${ }^{a)}$ Electronic mail: h.s.j.vanderzant@tudelft.nl.
Evaporation of the bridges is done on two different substrates: a $\mathrm{SiO}_{2}$ substrate or $\mathrm{SiO}_{2}$ substrate with a $\mathrm{Al} / \mathrm{Al}_{2} \mathrm{O}_{3}$ gate structure on top. The latter [see Fig. 1(a)] is used for three-terminal transport measurements. Contact to the nanowires is made through large Au contact pads, resulting in an initial wire resistance in the order of a few hundred ohms. Right before use, the samples were UV/ozone cleaned for 10 min. EM is done under ambient conditions while the temperature is regulated by a heater $(300-460 \mathrm{~K}$ range). The active feedback mechanism stops EM once a target conductance is reached in these experiments set at $300 \mu \mathrm{S}$. Using

(a)
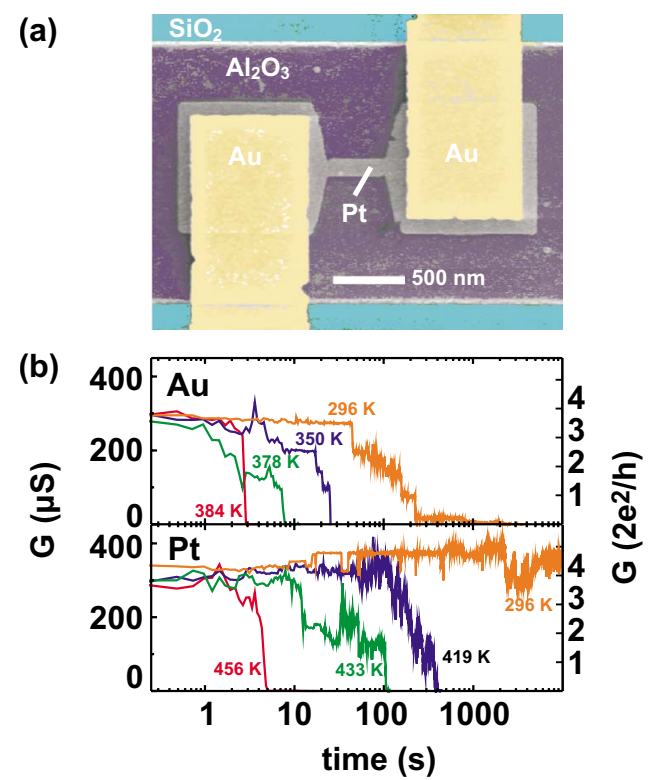

FIG. 1. (Color online) (a) Colorized scanning electron microscope image of the three-terminal device architecture before EM. The Pt nanowire (gray) is placed on top of the $\mathrm{Al} / \mathrm{Al}_{2} \mathrm{O}_{3}$ gate electrode (purple) which in turn is placed on top of the $\mathrm{SiO}_{2}$ substrate (blue). Contact to the nanowire is made through large Au contact pads (yellow). (b) (Top) Time evolution of the conductance for four $\mathrm{Au}$ nanowires initially narrowed to $300 \mu \mathrm{S}$ by $\mathrm{EM}$, and held at four different temperatures as indicated. (Bottom) Same as top but for four Pt nanowires. The measurements were performed on substrates without the $\mathrm{Al} / \mathrm{Al}_{2} \mathrm{O}_{3}$ gate structure. 


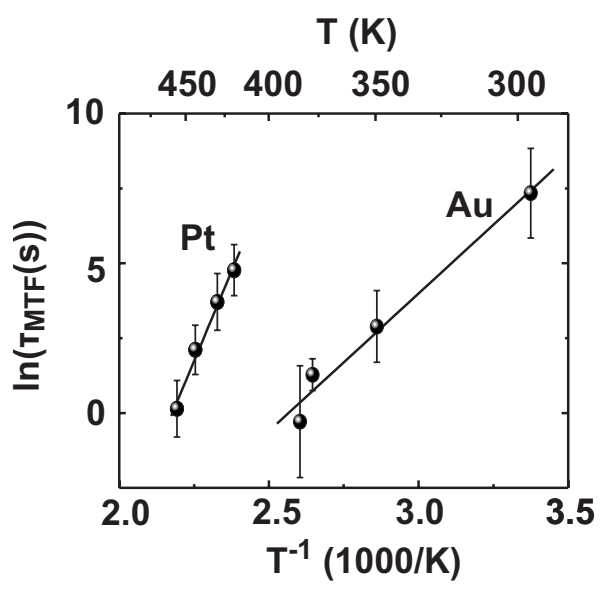

FIG. 2. The natural logarithm of the mean time to failure ( $\left.\tau_{\mathrm{MTF}}\right)$ vs inverse temperature for $\mathrm{Au}$ and $\mathrm{Pt}$ few-atom contacts electromigrated to $300 \mu \mathrm{S}$. Lines are linear fits to the data.

this feedback-controlled method, the yield for obtaining $300 \mu \mathrm{S}$ constrictions is $100 \%$.

After reaching the target conductance, the evolution of the conductance is determined by least-squares fitting of current-voltage characteristics between $\pm 100 \mathrm{mV}$ measured at $250 \mathrm{~ms}$ intervals. Control experiments in which the conductance was not monitored showed that these applied voltages did not influence the self-breaking. In each experiment, EM and self-breaking are performed at the same temperature. Figure 1(b) (top panel) shows selected time traces of the conductance of $\mathrm{Au}$ at different temperatures, representative of the overall behavior. As mentioned before, Au readily undergoes self-breaking at $300 \mathrm{~K}$. At elevated temperatures the self-breaking proceeds faster, indicating a temperature activated character of this process.

In contrast to $\mathrm{Au}, \mathrm{Pt}$ constrictions at $300 \mathrm{~K}$ are stable around the $300 \mu \mathrm{S}$ conductance value at which $\mathrm{EM}$ is stopped [orange curve in Fig. 1(b), bottom panel]. At temperatures above $420 \mathrm{~K}$ the constrictions start to show selfbreaking. However, whereas Au shows a nearly full selfbreaking percentage, for $\mathrm{Pt}$ it stays around $70 \%-80 \%$ even though the average time scale of self-breaking is decreasing over orders of magnitude with increasing temperature. Apparent from the Pt conductance traces when compared to the case of $\mathrm{Au}$ is the absence of well defined conductance plateaus. This can be attributed to the outer shell electrons, which in the case of Pt are located in the anisotropically shaped $d$-shell making the conductance more susceptible to changes in the atomic arrangement. ${ }^{8}$

In order to quantify the (in)stability of the few-atom contacts, we define the mean time to failure $\left(\tau_{\mathrm{MTF}}\right)$ as the average time it takes for the conductance to drop from $300 \mu \mathrm{S}$ down to a value below the conductance quantum $G_{0}$ $=2 e^{2} / h=77 \mu \mathrm{S}$. At four different temperatures, $\tau_{\mathrm{MTF}}$ is determined for both $\mathrm{Au}$ and $\mathrm{Pt}$ from a minimum of seven different time traces for each temperature, as the ones shown in Fig. 1. The traces of Pt few-atom contacts which do not undergo self-breaking are omitted. Despite the variation in the individual failure times, an exponential temperature dependence is observed for both materials, as shown in Fig. 2, which depicts $\ln \left(\tau_{\mathrm{MTF}}\right)$ as a function of $T^{-1}$. Assuming a temperature activated process we can write

$$
\tau_{\mathrm{MTF}}=A \exp \left(E_{a} / k_{B} T\right),
$$

in which $A$ is a constant, $E_{a}$ is the activation energy, $k_{B}$ is the Boltzmann constant, and $T$ is the temperature. From the slopes of the linear fits (drawn lines in Fig. 2) we find $E_{a}^{\mathrm{Au}}$ $=0.81 \pm 0.08 \mathrm{eV}$ and $E_{a}^{\mathrm{Pt}}=1.69 \pm 0.48 \mathrm{eV}$. The activation energy for $\mathrm{Au}$ is close to the literature value for the activation energy of grain boundary self-diffusion $(0.88 \mathrm{eV}){ }^{9}$ This observation suggests that atomic diffusion along grain boundaries is controlling the self-breaking in our few-atom constrictions. This assumption is supported by TEM studies of the self-breaking in Au few-atom contacts, in which a grain boundary was visible up to the point of breaking. ${ }^{10}$

We now turn to the Pt junctions and the main point of this letter. As Pt few-atom constrictions are stable at room temperature [see Fig. 1(b), bottom], junctions self-broken at elevated temperatures may be cooled down to form stable nanogaps. To characterize the three-terminal transport of the junctions in detail we use samples with a $\mathrm{Al} / \mathrm{Al}_{2} \mathrm{O}_{3}$ gate. EM is done consecutively on 32 devices on a chip at $430 \mathrm{~K}$ $\left(\tau_{\mathrm{MTF}} \approx 100 \mathrm{~s}\right) .{ }^{11}$ After self-breaking with a yield of approximately $70 \%$, the temperature is lowered by turning off the heater. Within a minute the temperature drops below $400 \mathrm{~K}$ and the gap stabilizes. Typically within one chip, this method yields a range of room-temperature nanogap resistances between $100 \mathrm{k} \Omega$ and infinite $(>100 \mathrm{G} \Omega)$. Before a detailed characterization by three-terminal measurements, the devices are once again subjected to a UV/ozone cleaning for $10 \mathrm{~min}$, after which they are transferred to the vacuum chamber of a low temperature probe station $\left(10^{-5} \mathrm{mbar}\right)$.

Current-voltage characteristics between $\pm 500 \mathrm{mV}$ of 172 self-broken junctions on eight different chips were measured at room temperature. Of these, 70 showed current levels below the detection limit of our setup (a few picoampere) within the applied voltage range; these junctions are disregarded in the rest of the paper. The remaining 102 junctions showed paraboliclike current-voltage curves and were cooled to $77 \mathrm{~K}$. No gate dependence in the current-voltage characteristics was found within the limits of the set by the leakage currents (which are lower than $50 \mathrm{pA}$ when applying gate voltages smaller than $\pm 2.5 \mathrm{~V}$ ). The absence of gatedependent features suggests that these junctions are free of metallic clusters. This observation is supported by comparing the current-voltage characteristics to a single-barrier tunneling model. We have fitted the current-voltage characteristics to the generalized formula for tunneling through a rectangular barrier with image forces included. ${ }^{12}$ The junction cross section is fixed at $100 \mathrm{~nm}^{2}$, based on a rough estimate of the actual size of the interface from TEM inspection on similar devices. Using the gap-size $(d)$ and barrier height $(\phi)$ as fit parameters, we find a good agreement to this model for all 102 junctions to the parabolic curvature. Fit parameters range from 3.0 to $3.9 \mathrm{eV}$ for $\phi$ and from 0.5 to $2.1 \mathrm{~nm}$ for $d$. Figure 3 shows two examples of such fits. Note that the fit-parameters are not very sensitive to the cross section. For instance when taking a cross section of $10 \mathrm{~nm}^{2}$ we find $\phi$ $=3.2 \mathrm{eV}$, and $d=1.32 \mathrm{~nm}$ for the sample displayed by the red curve in Fig. 3, instead of $3.4 \mathrm{eV}$ and $1.40 \mathrm{~nm}$ for a $100 \mathrm{~nm}^{2}$ cross section.

We find that the nanogaps show long-term stability in vacuum at room temperature, undergoing only minor changes in their resistance for periods up to 3 months. This stability was investigated in more detail by taking current- 


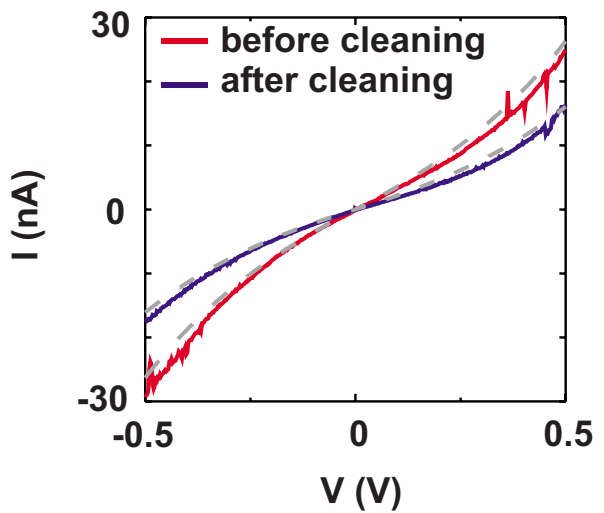

FIG. 3. (Color online) Current-voltage characteristic of a self-broken Pt junction at $77 \mathrm{~K}$ in vacuum (solid red line). After a UV/ozone cleaning step of $10 \mathrm{~min}$ at room temperature the current-voltage characteristic of the same sample was measured again at $77 \mathrm{~K}$ (solid blue line). A least-squares fit to the generalized formula for tunneling through a rectangular barrier with image charges included is displayed for both curves (dashed gray lines). For this fit, the junction cross section is fixed at $100 \mathrm{~nm}^{2}$. The fit-parameters are before cleaning: $d=1.40 \mathrm{~nm}$ and $\phi=3.4 \mathrm{eV}$, and after cleaning: $d=1.37 \mathrm{~nm}$ and $\phi=3.4 \mathrm{eV}$.

voltage characteristics of one of the junctions over a time span of $50 \mathrm{~h}$ with 2 min intervals (see Fig. 4, bottom panel). The low-bias resistance in this figure stays within one order of magnitude. This can be translated to atomic scale rearrangements on the sub-Ångström scale, as deduced from fitting each current-voltage characteristic to the above mentioned formula for tunneling (see Fig. 4, top panel, with $\phi$ fixed at $3.9 \mathrm{eV}$ and the junction cross section at $100 \mathrm{~nm}^{2}$ ). We also note the changes in resistance and therefore the electrode spacing stays within the same limits upon repeated thermal cycling between room temperature and $4 \mathrm{~K}$. Further-

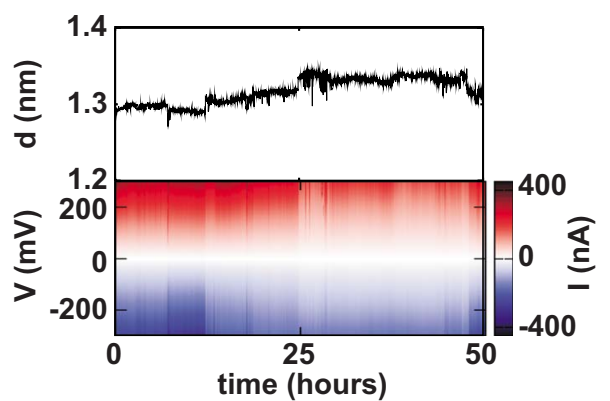

FIG. 4. (Color online) (Bottom) Two-dimensional color plot of the current (negative: blue, zero: white, and positive: red) as a function of voltage and time at room temperature. (Top) Electrode-separation $(d)$ from the fit of each individual current-voltage characteristic to the Simmons model for tunneling. For this fit, the junction cross section was fixed at $100 \mathrm{~nm}^{2}$ and the barrier height at $3.9 \mathrm{eV}$. more, this stability is maintained when taking the devices out of vacuum and performing a UV/ozone cleaning step of 10 min (see Fig. 3, blue curve).

In conclusion, we have presented a method for the fabrication of Pt-based nanogaps that are free of metallic clusters and that are stable at room temperature. The Pt-based gaps are interesting candidates for molecule deposition as their stability provides them with a number of advantages as compared to the traditional Au-based nanogaps. Molecule deposition can be performed after the gaps are formed, which avoids exposure of the molecules to the exceedingly high local temperatures which are generated during the EM process. ${ }^{13-15}$ Prior to molecule deposition, the bare gaps can be UV/ozone-cleaned and characterized in detail at low temperature. The presented fabrication method therefore opens up a route toward single molecule three-terminal transport at room temperature and possibly single-molecule device applications at ambient conditions.

Financial support was obtained from Stichting FOM (project 86) and from the EU FP7 program under the grant agreement "SINGLE" (project number 213609).

${ }^{1}$ H. Park, A. K. L. Lim, A. P. Alivisatos, J. Park, and P. L. McEuen, Appl. Phys. Lett. 75, 301 (1999).

${ }^{2}$ D. Natelson, L. H. Yu, J. W. Ciszek, Z. K. Keane, and J. M. Tour, Chem. Phys. 76, 267 (2005).

${ }^{3}$ D. R. Strachan, D. E. Smith, M. D. Fischbein, D. E. Johnston, B. S. Guiton, M. Drndic, D. A. Bonnel, and A. T. Johnson, Jr., Nano Lett. 6, 441 (2006).

${ }^{4}$ K. O’Neill, E. A. Osorio, and H. S. J. van der Zant, Appl. Phys. Lett. 90, 133109 (2007).

${ }^{5}$ R. Sordan, B. Balasubramanian, M. Burghard, and K. Kern, Appl. Phys. Lett. 87, 013106 (2005).

${ }^{6}$ H. S. J. van der Zant, Y.-V. Kervennic, M. Poot, K. O'Neill, Z. de Groot, H. B. Heersche, N. Stuhr-Hansen, T. Bjørnholm, D. Vanmaekelenbergh, C. A. van Walree, and L. W. Jenneskens, Faraday Discuss. 131, 347 (2006).

${ }^{7}$ D. R. Strachan, D. E. Smith, D. E. Johnston, T.-H. Park, M. J. Therien, D. A. Bonnel, and A. T. Johnson, Appl. Phys. Lett. 86, 043109 (2005).

${ }^{8}$ F. Pauly, M. Dreher, J. K. Viljas, M. Häfner, J. C. Cuevas, and P. Nielaba, Phys. Rev. B 74, 235106 (2006).

${ }^{9}$ D. Gupta, J. Appl. Phys. 44, 4455 (1973); No literature value for the grain boundary self diffusion was found for Pt.

${ }^{10}$ H. B. Heersche, G. Lientschnig, K. O’Neill, H. S. J. van der Zant, and H. W. Zandbergen, Appl. Phys. Lett. 91, 072107 (2007).

${ }^{11}$ The self-breaking rates of the constrictions on the $\mathrm{Al}_{2} \mathrm{O}_{3}$ substrate are comparable to those on $\mathrm{SiO}_{2}$.

${ }^{12}$ J. G. Simmons, J. Appl. Phys. 34, 1793 (1963).

${ }^{13}$ M. L. Trouwborst, S. J. van der Molen, and B. J. van Wees, J. Appl. Phys. 99, 114316 (2006).

${ }^{14}$ T. Taychatanapat, K. I. Bolotin, F. Kuemmeth, and D. C. Ralph, Nano Lett. 7, 652 (2007).

${ }^{15}$ D. R. Ward, N. J. Halas, and D. Natelson, Appl. Phys. Lett. 93, 213108 (2008) 